\title{
Intracoronary administration of anisodamine and nicorandil in individuals undergoing primary percutaneous coronary intervention for acute inferior myocardial infarction: A randomized factorial trial
}

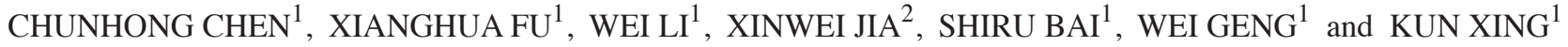 \\ ${ }^{1}$ Department of Cardiology, Second Hospital of Hebei Medical University, Shijiazhuang, Hebei 050000; \\ ${ }^{2}$ Department of Cardiology, The Affiliated Hospital of Hebei University, Baoding, Hebei 071000, P.R. China
}

Received November 6, 2014; Accepted May 29, 2015

DOI: $10.3892 /$ etm.2015.2623

\begin{abstract}
In acute inferior myocardial infarction (AIMI), numerous conventional drugs that are used to improve the myocardial microcirculation can significantly reduce blood pressure (BP) and coronary perfusion pressure, aggravate bradyarrhythmia and cause a deterioration in the hemodynamic state of the whole body, which greatly limits the application of these drugs in clinical settings. The aim of the present study was to assess the effect of anisodamine and nicorandil regimens on the prevention of no-reflow (NR) and the amelioration of myocardial reperfusion in patients with AIMI undergoing primary percutaneous coronary intervention (PCI). A total of 104 consecutive patients with AIMI were included in this study and randomly assigned to one of four groups: A (control group), B (anisodamine group), C (nicorandil group) and D (anisodamine and nicorandil group). Patients underwent PCI via transradial artery access and the angiographic results were evaluated. Coronary diastolic BP (DBP) and systolic and mean BPs were measured by invasive catheterization. The primary end-point was a post-PCI Thrombolysis In Myocardial Infarction (TIMI) myocardial perfusion grade (TMPG) of 3. Composite end-points (mortality + new MI + target vessel revascularization) were evaluated during the hospital stay and 30 days after discharge. Following the procedure, the proportion of patients achieving TMPG 3 was significantly higher in group $\mathrm{D}$ than that in the other groups $(\mathrm{P}=0.014)$; furthermore, the incidence of a postprocedural TIMI score of 3 was the highest in group D. Three days after the procedure, the peak creatine kinase-MB and cardiac troponin I levels were the lowest and the left ventricular ejection fraction was the highest
\end{abstract}

Correspondence to: Mr. Xianghua Fu, Department of Cardiology, Second Hospital of Hebei Medical University, 215 Hepingxi Road, Shijiazhuang, Hebei 050000, P.R. China

E-mail: xianghuafuh@163.com

Key words: anisodamine, nicorandil, no reflow, acute inferior myocardial infarction, primary percutaneous coronary intervention in group D. A thrombus score of $3 / 4$ and low DBP were the independent risk factors for poor myocardial reperfusion (expressed as TMPG <3), while $2 \mathrm{mg}$ anisodamine plus $2 \mathrm{mg}$ nicorandil prior to PCI was protective for optimal myocardial reperfusion following the procedure. The combination of anisodamine and nicorandil can effectively ameliorate myocardial reperfusion and protect cardiac function in patients with AIMI undergoing primary PCI.

\section{Introduction}

The no-reflow (NR) phenomenon, an important manifestation of disordered myocardial microcirculation, is the failure of the blood to reperfuse an ischemic area following the removal of the physical obstruction by primary percutaneous coronary intervention (PCI) or thrombolysis $(1,2)$. The incidence and extent of NR strongly predict adverse clinical outcomes, including persistent contractile dysfunction of the left ventricle, malignant arrhythmias and cardiac death (3).

Acute inferior myocardial infarction (AIMI), in which the right coronary artery (RCA) is often the infarct-related artery (IRA), is usually indicative of two characteristics: i) A heavier thrombus burden, which significantly increases the risk of NR following primary PCI (4); and ii) unstable hemodynamic states, such as hypotension, bradycardia and high vagus tone, which can result in circulatory collapse, reperfusion injury and serious arrhythmias, including severe atrioventricular block, during primary PCI (5).

Anisodamine, a drug with multiple pharmacological effects, can maintain the stability of the hemodynamic states and increase heart rate (HR), blood pressure (BP), coronary perfusion pressure and heart inotropy, making it suitable for AIMI. Anisodamine is also an M-receptor blocker and can dilate the small vessels and improve the microcirculation (6). Our previous studies found that intracoronary administration of anisodamine was effective in reversing the NR following primary PCI (6-9).

Nicorandil maintains the activation of adenosine triphosphate (ATP)-sensitive $\mathrm{K}^{+}$channels and induces nitric oxide production in an identical manner to that of nitrates, resulting in the dilatation of the coronary microcirculation, ischemic 
preconditioning, anti-arrhythmic effects and a reduction in reperfusion injury. Intravenous nicorandil administration in patients with acute MI (AMI) undergoing PCI has been shown to effectively improve left ventricular systolic function and reduce NR and slow flow (10-12); however, to the best of our knowledge, no studies have investigated the effect of intracoronary nicorandil and anisodamine administration in patients with AIMI and, therefore, the optimal dosage, administration method and safety of the combination therapy remain unknown. Based on this, the objective of the present study was to assess the effect of anisodamine and nicorandil regimens on the prevention of NR and the amelioration of myocardial reperfusion in patients with AIMI undergoing primary PCI.

\section{Materials and methods}

Study population inclusion criteria. Between September 2011 and January 2014, a total of 115 consecutive patients with AIMI who were admitted within $12 \mathrm{~h}$ of symptom onset and treated through primary PCI were enrolled into this open-label, randomized, controlled study. Eleven patients were excluded subsequently due to a lack of file information and an emergency status requiring surgical intervention. Consequently, the population of this study consisted of 104 patients.

Patients were eligible if they fulfilled the following criteria: i) Ischemic chest pain lasting for $\geq 20 \mathrm{~min}$, which could not be relieved by oral nitrates; and ii) clear AIMI-related changes in the electrocardiogram (ECG) during chest pain, i.e. new ST-segment elevation with the cut-off points $\geq 1 \mathrm{~mm}$ in $\geq 2$ standard leads or $\geq 2 \mathrm{~mm}$ in $\geq 2$ contiguous precordial leads with or without the elevation of cardiac enzymes.

Study population exclusion criteria. Patients were excluded if one of the following characteristics was present: i) Cardiogenic shock (Killip class IV); ii) tachycardia (HR $\geq 100$ bpm); iii) known allergy to every essential drug; iv) bleeding history; v) hepatic dysfunction; vi) renal dysfunction (creatinine $>12 \mathrm{mg} / \mathrm{dl}$ ); vii) thrombolysis; viii) requirement for coronary artery bypass grafting (CABG); ix) contraindication to antiplatelet and anticoagulation therapy; and $\mathrm{x}$ ) previous MI. The study was approved by the Ethics Committee of the Second Hospital of Hebei Medical University (Shijiazhuang, China) and the Affiliated Hospital of Hebei University (Baoding, China), and written informed consent was obtained from all patients.

Doseandtiming ofanisodamine andnicorandiladministration . Subjects were immediately transferred to the cathlab to undergo emergency PCI (door-to-balloon $\leq 90$ mins). The study population was divided into four groups ( $\mathrm{n}=26$ per group): A (control group, treated with PCI only), B (anisodamine group, treated with intracoronary anisodamine), C (nicorandil group, treated with intracoronary nicorandil) and D (anisodamine and nicorandil group, treated with intracoronary nicorandil and anisodamine). Patients received $2 \mathrm{mg}$ anisodamine (Minsheng Pharmaceutical Group Co. Ltd., Hangzhou, China) and $2 \mathrm{mg}$ nicorandil (Sihuan Kebao Pharmaceutical Group Co. Ltd., Beijing, China) via intracoronary administration. The optimal timing of drug administration was as follows: i) If initial Thrombolysis In Myocardial Infarction [TIMI (13)] grade $>0$, drugs can be administered into the coronary artery through the guide catheter; or ii) if initial TIMI grade $=0$, the guidewire should be advanced through the total occlusion to the distal site of the IRA. The anterior flow of the IRA will then recover, which means that the TIMI grade will be $>0$ so drugs can then be administered into the coronary artery through the guide catheter.

The administration of other medications was performed according to the current best clinical practice: Aspirin, loading dose of $300 \mathrm{mg}$ and then $100 \mathrm{mg}$ once daily (qd); clopidogrel, loading dose of $600 \mathrm{mg}$ and then $75 \mathrm{mg} \mathrm{qd}$; heparin, 40-70 U/kg (activated clotting time >200 sec); low-molecular-weight heparin; angiotensin-converting enzyme inhibitors/angiotensin receptor blockers [systolic BP (SBP) >120 mmHg]; $\beta$-blockers (HR >60 bpm) and statins. Tirofiban administration was under the careful discretion of the interventional cardiologists.

Coronary angiography and other examinations. Patients underwent PCI via transradial artery access. The initial TIMI, final TIMI/corrected TIMI frame count and TIMI myocardial perfusion grade [TMPG (14)] were evaluated by two cardiologists blinded to the clinical status of the patient and the treatment modality.

Coronary SBP, diastolic BP (DBP) and mean BP were measured by invasive catheterization before and 1,5 and $10 \mathrm{~min}$ after anisodamine and nicorandil administration. Infarct size was estimated by peak levels of creatine kinase-MB (CK-MB) and troponin I (cTnI), which were determined before and every $4 \mathrm{~h}$ after the procedure using a Coulter LH 780 Hematology Analyzer (Beckman Coulter Ireland Inc., Galway, Ireland).

A 12-lead ECG was recorded both on admission and $90 \mathrm{~min}$ after PCI. ST-segment elevation was recorded $20 \mathrm{msec}$ after the J point. The sum ST-segment elevation was calculated in leads II, III and aVF for inferior infarction. A decrease in the sum ST-segment elevation by $\geq 70 \%$ was categorized as complete ST-segment resolution (STR) (15) and used as an indirect measure of myocardial reperfusion following PCI (16).

Primary end-point. The primary end point was a TMPG of 3 (17) following the procedure.

Major adverse cardiovascular events (MACEs). The MACEs included i) all-cause mortality; ii) new MI, as indicated by the onset of recurrent ischemic chest pain lasting $\geq 30 \mathrm{~min}$ with new ST-T segment changes of $\geq 24 \mathrm{~h}$ duration or new pathologic Q waves ( $\geq 2$ leads) and an elevation in serum $\mathrm{CK}$ levels to >2-fold the normal upper limit or an elevated CK-MB fraction value; iii) target vessel revascularization (TVR) for recurrent ischemia or acute stent occlusion, including repeat PCI or CABG. Composite end-points, i.e. mortality + new MI + TVR) were evaluated during the hospital stay and 30 days after discharge.

Statistical analysis. In the PCI era, a reasonable estimate of the proportion of patients achieving optimal myocardial reperfusion (TMPG 3) among patients without cardiogenic shock undergoing PCI is $\sim 50 \%$ (1). It is speculated that the proportion of patients achieving a postprocedural TMPG of 3 can reach $90 \%$ following anisodamine and nicorandil administra- 
Table I. Main demographic/clinical features.

\begin{tabular}{|c|c|c|c|c|c|c|}
\hline Variables & Group A & Group B & Group C & Group D & $\mathrm{F} / \chi^{2}$ & P-value \\
\hline Age, years & $59.8 \pm 4.8$ & $58.9 \pm 5.1$ & $57.6 \pm 4.7$ & $58.6 \pm 4.5$ & 0.223 & 0.889 \\
\hline Male, n (\%) & $19(73.1)$ & $17(65.4)$ & $18(69.2)$ & $21(80.8)$ & 1.619 & 0.647 \\
\hline $\mathrm{BMI}, \mathrm{kg} / \mathrm{m}^{2}$ & $20.5 \pm 2.6$ & $21.2 \pm 2.5$ & $20.9 \pm 2.0$ & $19.9 \pm 1.8$ & 0.608 & 0.611 \\
\hline Hypertension, n (\%) & $12(46.2)$ & $14(53.8)$ & $10(38.5)$ & $11(42.3)$ & 1.228 & 0.727 \\
\hline Diabetes, n (\%) & $8(30.8)$ & $7(26.9)$ & $5(19.2)$ & $9(34.6)$ & 1.409 & 0.712 \\
\hline Hyperlipidemia, n (\%) & $6(23.1)$ & $8(30.8)$ & $5(19.2)$ & $6(23.1)$ & 0.969 & 0.805 \\
\hline Current smokers, n (\%) & $17(65.4)$ & $16(61.5)$ & $15(57.7)$ & $20(76.9)$ & 1.782 & 0.604 \\
\hline Family history of CHD, n (\%) & $6(23.1)$ & $7(26.9)$ & $4(15.4)$ & $9(34.6)$ & 2.156 & 0.526 \\
\hline Preinfarction angina, n (\%) & $5(19.2)$ & $4(15.4)$ & $8(30.8)$ & $5(19.2)$ & 2.026 & 0.544 \\
\hline Previous MI, n (\%) & $3(11.5)$ & $3(11.5)$ & $4(15.4)$ & $3(11.5)$ & 0.335 & 0.934 \\
\hline Previous PCI, n (\%) & $2(7.7)$ & $0(0.0)$ & $2(7.7)$ & $4(15.4)$ & 2.837 & 0.412 \\
\hline Killip class $>1, \mathrm{n}(\%)$ & $4(15.4)$ & $5(19.2)$ & 7 (26.9) & $6(23.1)$ & 0.754 & 0.826 \\
\hline $\mathrm{SCr}, \mu \mathrm{moI} / 1$ & $82.1 \pm 12.5$ & $82.9 \pm 17.1$ & $83.1 \pm 12.8$ & $84.5 \pm 17.6$ & 0.311 & 0.749 \\
\hline \multicolumn{7}{|l|}{ Medications, n (\%) } \\
\hline Aspirin & $26(100)$ & $26(100)$ & $26(100)$ & $26(100)$ & - & - \\
\hline Clopidogrel & $26(100)$ & $26(100)$ & $26(100)$ & $26(100)$ & - & - \\
\hline Statin & $26(100)$ & $26(100)$ & $26(100)$ & $26(100)$ & - & - \\
\hline ACEI/ARB & $18(69.2)$ & $20(76.9)$ & $21(80.8)$ & $16(61.5)$ & 2.727 & 0.428 \\
\hline$\beta$-blockers & 7 (26.9) & $10(38.5)$ & $9(34.6)$ & $11(42.3)$ & 0.824 & 0.821 \\
\hline Tirofiban & $2(7.7)$ & $4(15.4)$ & $2(7.7)$ & 3 (11.5) & 1.594 & 0.653 \\
\hline
\end{tabular}

n=26 per group. Group A, control; group B, 2 mg anisodamine; group C, 2 mg nicorandil; group D, 2 mg anisodamine and 2 mg nicorandil; BMI, body mass index; CHD, coronary heart disease; MI, myocardial infarction; PCI, percutaneous coronary intervention; SCr, serum creatinine; ACEI, angiotensin-converting enzyme inhibitors; ARB, angiotensin receptor blockers.

tion. Accordingly, $\geq 26$ patients per group were required for the power of the test set at 0.8 and the statistical significance level (two-sided) at 0.025 .

Continuous variables are expressed as the mean \pm standard deviation, and categorical variables are presented as percentages. Continuous variables were compared using analysis of variance (ANOVA), and proportions were compared using the $\chi^{2}$ or Fisher's exact tests. Multivariate logistic regression analysis was used to explore the possible factors associated with the optimal myocardial reperfusion (TMPG 3). For the peak CK-MB and left ventricular ejection fraction (LVEF), factorial design ANOVA was applied to elucidate the main effects and interactions of the two drugs. A Student-Newman-Keuls (q) test was used for post hoc analysis if there was a significant difference among the groups. Two-sided P-values of $<0.05$ were considered statistically significant. All calculations were computed with the aid of SPSS statistical software (version 16.0; SPSS, Inc., Chicago, IL, USA).

\section{Results}

Main demographic and clinical characteristics. No significant differences in age, gender, past medical histories (hypertension, diabetes, current smokers, previous angina/MI/PCI) and basic medication use were found among the four groups (Table I).
Procedural characteristics. In the four groups, the IRA was predominantly the RCA followed by the left circumflex; in excess of one-half of the IRAs had a thrombus score of 3/4 (18). Following the procedure, the proportion of TMPG 3 cases was significantly higher in group $\mathrm{D}$ than that in other groups $(\mathrm{P}=0.014)$; furthermore, the proportions of postprocedural TIMI 3 and complete STR cases were the highest in group D, although no significant differences were found $(\mathrm{P}=0.067$ and 0.052 , respectively) (Table II).

Main clinical index and follow up. Three days after the procedure, the peak CK-MB and cTnI levels were the lowest and the LVEF was highest in group D. Nicorandil had no obvious effect on the vital signs during and following the procedure. Anisodamine elevated the BP and HR. One patient in group B developed paroxysmal atrial tachycardia; however, no other severe tachyarrhythmias, such as ventricular tachycardia or ventricular fibrillation, were observed during or following the procedure. The incidence of MACEs during hospitalization and the 30-day follow-up after discharge did not differ among the four groups (Table III and Fig. 1).

Predictors of TMPG 3 by multivariate logistic analysis. A number of variables associated with the TMPG following the procedures were enrolled into the multivariate logistic regression model. The result showed that a thrombus score of 
Table II. Procedural characteristics.

\begin{tabular}{|c|c|c|c|c|c|c|}
\hline Variables & Group A & Group B & Group C & Group D & $\mathrm{F} / \chi^{2}$ & P-value \\
\hline Onset to balloon, $\mathrm{h}$ & $6.8 \pm 1.6$ & $7.2 \pm 1.7$ & $7.5 \pm 1.6$ & $6.7 \pm 2.0$ & 1.952 & 0.132 \\
\hline Door to balloon, min & $65.8 \pm 17.5$ & $73.5 \pm 20.81$ & $60.1 \pm 18.2$ & $65.1 \pm 20.6$ & 0.975 & 0.420 \\
\hline \multicolumn{7}{|l|}{ IRA, n (\%) } \\
\hline $\mathrm{RCA}$ & $20(76.9)$ & $16(61.5)$ & $22(84.6)$ & $23(88.5)$ & 3.855 & 0.290 \\
\hline $\mathrm{LCX}$ & $8(30.8)$ & $11(42.3)$ & 7 (26.9) & $6(23.1)$ & 2.957 & 0.429 \\
\hline LAD & $0(0.0)$ & $1(3.85)$ & $0(0.0)$ & $0(0.0)$ & 2.935 & 0.420 \\
\hline \multicolumn{7}{|l|}{ Thrombus scores, n (\%) } \\
\hline 0 & $5(19.2)$ & $2(7.7)$ & $4(15.4)$ & $3(11.5)$ & 0.985 & 0.872 \\
\hline $1-2$ & $10(38.5)$ & $8(30.8)$ & $8(30.8)$ & $12(46.2)$ & 1.219 & 0.772 \\
\hline $3-4$ & $17(65.4)$ & $15(57.7)$ & $16(61.5)$ & $16(61.5)$ & 1.023 & 0.795 \\
\hline TIMI 3 pre-PCI, n (\%) & $3(11.5)$ & $2(7.7)$ & $2(7.7)$ & $2(11.5)$ & 1.013 & 1.271 \\
\hline Mean TIMI score pre-PCI, n (\%) & $0.83 \pm 1.61$ & $0.65 \pm 1.02$ & $0.58 \pm 0.78$ & $0.50 \pm 0.68$ & 0.320 & 0.765 \\
\hline TIMI 3 post-PCI, n (\%) & $20(76.9)$ & $22(84.6)$ & $21(80.8)$ & $24(92.3)$ & 7.657 & 0.067 \\
\hline Mean TIMI score post-PCI, n (\%) & $2.79 \pm 0.57$ & $2.82 \pm 0.19$ & $2.76 \pm 0.50$ & $3.00 \pm 0.00$ & 2.016 & 0.122 \\
\hline TMPG 3 post-PCI, n (\%) & $17(65.4)$ & $21(80.8)$ & $20(76.9)$ & $23(88.5)$ & 1.065 & 0.014 \\
\hline Mean TMPG post-PCI, n (\%) & $2.58 \pm 0.78$ & $2.80 \pm 0.52$ & $3.05 \pm 0.49$ & $3.12 \pm 0.00$ & 2.535 & 0.073 \\
\hline Stents per patient, $\mathrm{n}(\%)$ & $1.5 \pm 0.9$ & $1.7 \pm 1.1$ & $1.6 \pm 0.8$ & $1.6 \pm 0.7$ & 0.312 & 0.764 \\
\hline Complete STR, n (\%) & $17(65.4)$ & $21(80.8)$ & $21(80.8)$ & $25(96.2)$ & 7.631 & 0.052 \\
\hline
\end{tabular}

n=26 per group. Group A, control; group B, 2 mg anisodamine; group C, 2 mg nicorandi; group D, 2 mg anisodamine and 2 mg nicorandil; IRA, infarct-related artery; RCA, right coronary artery; LCX, left circumflex; LAD, left anterior descending; TIMI, Thrombolysis In Myocardial Infarction; TMPG, TIMI myocardial perfusion grade; STR, ST-segment resolution.
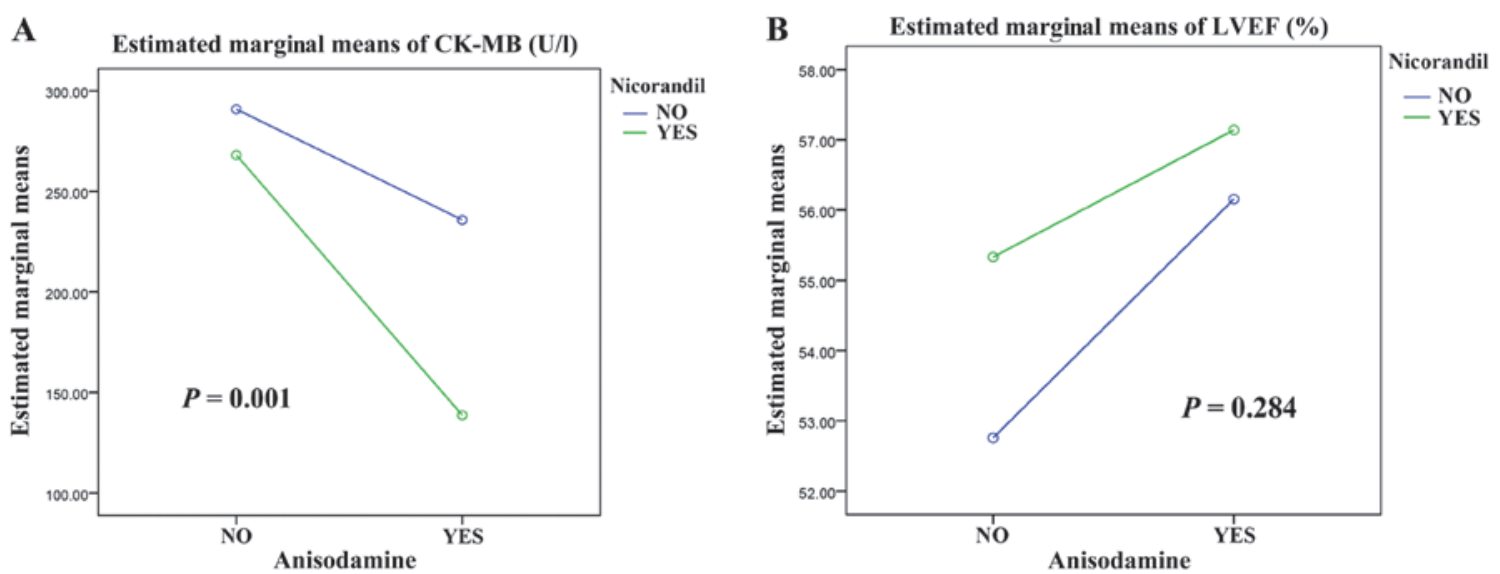

Figure 1. Main clinical index and follow-up. Interactions of anisodamine and nicorandil for (A) peak CK-MB and (B) LVEF. CK-MB, creatine kinase-MB; LVEF, left ventricular ejection fraction.

3/4 and low DBP prior to anisodamine and nicorandil administration were the independent risk factors for poor myocardial reperfusion (expressed as TMPG $<3$ ), while $2 \mathrm{mg}$ anisodamine and $2 \mathrm{mg}$ nicorandil prior to PCI were associated with optimal myocardial reperfusion following the procedure (Table IV).

\section{Discussion}

As an important manifestation of disordered myocardial microcirculation, NR is a multifactorial phenomenon associ- ated with numerous pathological changes that eventually lead to microvascular obstruction and impaired myocardial perfusion $(14,19,20)$. The main factors that contribute to NR are as follows: i) Damage to the microvascular structure by endothelial and myocardial cell edema, which leads to microcirculatory compression and cell ischemia; ii) ischemia-reperfusion injury-induced endothelial swelling, interstitial and intracellular edema and intraluminal microthrombi (14); iii) microvascular spasms caused by a dysfunctional endothelium and potent vasoconstrictors (sero- 
Table III. Main clinical index and follow up.

\begin{tabular}{|c|c|c|c|c|c|c|}
\hline Variables & Group A & Group B & Group C & Group D & $\mathrm{F} / \chi^{2}$ & P-value \\
\hline Peak CK-MB, U/1 & $281.9 \pm 56.5$ & $243.7 \pm 58.1$ & $258.6 \pm 65.5$ & $141.9 \pm 42.3$ & 39.328 & $<0.001$ \\
\hline Peak cTnI, ng/ml & $64.8 \pm 19.8$ & $56.9 \pm 18.9$ & $61.4 \pm 21.1$ & $50.2 \pm 18.1$ & 4.875 & 0.005 \\
\hline LVEF, \% & $51.9 \pm 4.5$ & $56.8 \pm 4.7$ & $49.3 \pm 3.8$ & $58.1 \pm 3.8$ & 6.875 & $<0.001$ \\
\hline \multicolumn{7}{|l|}{$\mathrm{BP}$ and HR before treatment } \\
\hline $\mathrm{SBP}, \mathrm{mmHg}$ & $108.1 \pm 11.7$ & $106.3 \pm 13.3$ & $104.3 \pm 13.6$ & $105.7 \pm 12.5$ & 0.039 & 0.971 \\
\hline $\mathrm{DBP}, \mathrm{mmHg}$ & $64.1 \pm 8.7$ & $61.9 \pm 8.1$ & $63.7 \pm 9.8$ & $62.8 \pm 7.3$ & 0.835 & 0.478 \\
\hline $\mathrm{MBP}, \mathrm{mmHg}$ & $78.8 \pm 6.0$ & $76.7 \pm 7.2$ & $77.2 \pm 8.2$ & $77.1 \pm 2.3$ & 0.393 & 0.741 \\
\hline $\mathrm{HR}, \mathrm{bpm}$ & $51.8 \pm 9.4$ & $52.6 \pm 5.5$ & $52.5 \pm 6.0$ & $58.3 \pm 9.2$ & 0.482 & 0.701 \\
\hline \multicolumn{7}{|l|}{$\mathrm{BP}_{\max }$ and $\mathrm{HR}_{\max }$ after treatment } \\
\hline $\mathrm{SBP}, \mathrm{mmHg}$ & $114.1 \pm 10.5$ & $129.5 \pm 11.8$ & $116.4 \pm 18.4$ & $130.6 \pm 17.2$ & 3.547 & 0.026 \\
\hline $\mathrm{DBP}, \mathrm{mmHg}$ & $71.0 \pm 12.8$ & $76.2 \pm 18.2$ & $69.4 \pm 4.9$ & $78.4 \pm 2.5$ & 2.978 & 0.035 \\
\hline $\mathrm{MBP}, \mathrm{mmHg}$ & $85.4 \pm 8.9$ & $94.0 \pm 7.3$ & $85.1 \pm 6.3$ & $95.8 \pm 3.0$ & 6.005 & 0.011 \\
\hline $\mathrm{HR}, \mathrm{bpm}$ & $62.6 \pm 9.1$ & $71.6 \pm 9.1$ & $63.5 \pm 7.3$ & $72.2 \pm 7.3$ & 2.035 & 0.213 \\
\hline \multicolumn{7}{|l|}{ In-hospital MACEs, n (\%) } \\
\hline Total & $0(0.0)$ & $0(0.0)$ & $0(0.0)$ & $0(0.0)$ & - & - \\
\hline Mortality & $0(0.0)$ & $0(0.0)$ & $0(0.0)$ & $0(0.0)$ & & \\
\hline New MI & $0(0.0)$ & $0(0.0)$ & $0(0.0)$ & $0(0.0)$ & & \\
\hline TVR & $0(0.0)$ & $0(0.0)$ & $0(0.0)$ & $0(0.0)$ & & \\
\hline MACEs during 30-day follow-up, n (\%) & & & & & 4.371 & 0.362 \\
\hline Total & $3(11.5)$ & $1(3.8)$ & $2(7.7)$ & $0(0.0)$ & & \\
\hline Mortality & $1(3.8)$ & $0(0.0)$ & $1(3.8)$ & $0(0.0)$ & & \\
\hline New MI & $1(3.8)$ & $1(3.8)$ & $0(0.0)$ & $0(0.0)$ & & \\
\hline TVR & $1(3.8)$ & $0(0.0)$ & $1(3.8)$ & $0(0.0)$ & & \\
\hline PAT, n (\%) & $0(0.0)$ & $1(3.8)$ & $0(0.0)$ & $0(0.0)$ & 2.499 & 0.398 \\
\hline VT/VF, n (\%) & $0(0.0)$ & $0(0.0)$ & $0(0.0)$ & $0(0.0)$ & - & - \\
\hline
\end{tabular}

$\mathrm{n}=26$ per group. Group A, control; group B, $2 \mathrm{mg}$ anisodamine; group C, $2 \mathrm{mg}$ nicorandil; group D, 2 mg anisodamine and 2 mg nicorandil; CK-MB, creatine kinase-MB; cTnI, cardiac troponin I; LVEF, left ventricular ejection fraction; SBP, systolic blood pressure; DBP, diastolic blood pressure; MBP, mean blood pressure; HR, heart rate; MACE, major adverse cardiovascular event; MI, myocardial infarction;TVR, target vessel revascularization; VT, ventricular tachycardia; VF, ventricular fibrillation; PAT, paroxysmal atrial tachycardia.

Table IV. Predictors of TIMI myocardial perfusion grade 3 by multivariate logistic analysis.

\begin{tabular}{lccc}
\hline Variables & P-value & OR & $95 \%$ CI \\
\hline Age & 0.987 & 1.008 & $0.870-1.165$ \\
Male & 0.959 & 0.968 & $0.249-3.671$ \\
Thrombus scores 3/4 & 0.037 & 0.177 & $0.029-0.908$ \\
TIMI grade 3 pre-PCI & 0.521 & 2.254 & $0.178-26.024$ \\
DBP before treatment & 0.048 & 1.075 & $1.002-1.169$ \\
Anisodamine (2 mg) & 0.040 & 3.483 & $1.042-11.549$ \\
Nicorandil (2 mg) & 0.047 & 2.501 & $0.739-8.346$ \\
\hline
\end{tabular}

TIMI, Thrombolysis In Myocardial Infarction; PCI, percutaneous coronary intervention; DBP, diastolic blood pressure; OR, odds ratio; CI, confidence interval.

tonin, angiotensin II, endothelin-1) (21); iv) enhanced tissue damage due to mechanical obstruction of leukocytes and their released products; v) obstruction of the microcirculation by microemboli consisting of thrombus fragments, blood cell aggregates, platelet plugs and atherosclerotic debris (22); and vi) extrinsic coagulation pathway activation (22).

TIMI grades reflect the epicardial blood perfusion, while the TMPG reflects myocardial tissue perfusion. Patients 
with NR, even those with TIMI score 3 but TMPG $<2$, are likely to suffer from adverse cardiovascular complications and poor clinical prognosis; therefore, while epicardial blood perfusion should be monitored, more attention should be paid to myocardial tissue perfusion. Blood perfusion without tissue perfusion is equal to no blood. The prevention of NR is crucial due to the fact that, to date, treatments are still lacking.

In the 1970s, anisodamine was used in the treatment of bacteremic shock (23) which was, in essence, a microcirculation disorder. NR is a myocardial microcirculation disorder, which is similar to bacteremic shock. It is therefore reasonable that anisodamine is used for the improvement of myocardial microcirculation.

The mechanisms of anisodamine in the prevention of NR and improvement of myocardial microcirculation are suggested to be multifactorial. Anisodamine relieves the spasms of conductive arteries, prearterioles and arterioles, dredges the coronary microcirculation and restores the autonomic microvascular rhythm of tide-like perfusion, which is destroyed by NR. Furthermore, anisodamine acts to increase the coronary perfusion pressure by increasing the BP (particularly the DBP and mean BP) and HR, which is beneficial for the correction of NR and the improvement of coronary microcirculation. In addition, anisodamine inhibits the acetylcholine receptor and regulates the rebalance between the sympathetic and vagus nervous systems, corrects hypotension and bradycardia and maintains hemodynamic stabilization. To a certain extent, anisodamine has a similar role to a calcium channel antagonist; thus, it can prevent intracellular calcium overload and attenuate microcirculatory spasms (6). By inhibiting lipid superoxidation and oxygen free radical formation, anisodamine can additionally lessen endothelial cell injury (24). Finally, anisodamine can alleviate the oppression of myocardial swelling on the microcirculation so as to improve the coronary antegrade blood flow (25). The present study showed that the intracoronary administration of $2 \mathrm{mg}$ anisodamine was safe; with the exception of one patient developing paroxysmal atrial tachycardia, no other severe tachyarrhythmias were observed during or following the procedure. Anisodamine elevated $\mathrm{BP}$ and HR, increased the coronary perfusion pressure and ameliorated the myocardial reperfusion, thus enhancing the clinical prognosis.

Intravenous nicorandil acts to dilate the coronary artery microvasculature, induces antiarrhythmic effects and reduces reperfusion injury via ATP-sensitive $\mathrm{K}^{+}$channels A previous study reported that, in AMI, intravenous nicorandil administration reduced injury to the myocardium and improved heart function, as shown by quantitative thallium single-photon emission computerized tomography analysis and echocardiographic regional wall motion scoring, respectively (26). In a different study, nicorandil administration in patients with AMI led to reductions in NR (nicorandil group $15 \%$ vs. control group 33\%), ventricular arrhythmia, heart failure and in-hospital mortality (27). A number of explanations have been suggested for these effects. First, the suppression of the $\mathrm{Na}^{+} / \mathrm{Ca}^{2+}$ exchange and the reduction in the myocardial calcium levels following the activation of ATP-sensitive $\mathrm{K}^{+}$channels may minimize the injury to the myocardium. Secondly, nicorandil exerts a protective effect on the heart by improving coronary blood flow: Nicorandil reduces neutrophil activation, which can suppress the inflammatory reactions and thereby reduce the resistance of the microvasculature. Finally, by dilating microvessels with a diameter of $<100 \mu \mathrm{m}$, nicorandil can decrease the preload of the heart and improve myocardial microcirculation (28). A previous study examined an intracoronary nicorandil group, a combined intravenous and intracoronary group and a control group in a series of AMIs; compared with the other two groups, the intracoronary nicorandil group exhibited reductions in reperfusion arrhythmia, NR, slow flow and chest pain (composite end-point $13 \%$ in the intracoronary group vs. $33 \%$ in the control group), although the differences were not significant (11); however, the dosage of intracoronary nicorandil used in the study was low $(0.5 \mathrm{mg}$ per dose, maximum dose 1-2 mg). In the present study, intracoronary nicorandil administration in AIMI led to similar results to those observed for intravenous nicorandil administration (15). The present study showed that intracoronary nicorandil administration ( $2 \mathrm{mg}$ ) was safe and effective in improving post-PCI TIMI and TMPG.

The results of this study showed that anisodamine and nicorandil could improve TIMI blood flow and TMPG, promote complete STR following the procedure, notably decrease the post-procedural peak levels of CK-MB and cTnI (indicating that the size of MI was decreased) and significantly improve the cardiac function (reflected by LVEF). This study demonstrated that anisodamine and nicorandil exhibited cooperative interactions and that the efficacy of anisodamine and nicorandil was superior to that of the other regimens. The data from this study also showed that a thrombus score of 3/4 and low DBP prior to treatment were the independent risk factors for poor myocardial reperfusion (expressed as TMPG <3), while anisodamine and nicorandil (2 mg each) prior to PCI were associated with optimal myocardial reperfusion following the procedure. In conclusion, a combinatorial therapy of anisodamine and nicorandil is an effective means of ameliorating myocardial reperfusion and protecting cardiac function in patients with AIMI undergoing primary PCI.

\section{References}

1. Niccoli G, Burzotta F, Galiuto L and Crea F: Myocardial no-reflow in humans. J Am Coll Cardiol 54: 281-292, 2009.

2. Zhao YJ, Fu XH, Ma XX, Wang DY, Dong QL, Wang YB, Li W, Xing $\mathrm{K}, \mathrm{Gu} \mathrm{XS}$ and Jiang YF: Intracoronary fixed dose of nitroprusside via thrombus aspiration catheter for the prevention of the no-reflow phenomenon following primary percutaneous coronary intervention in acute myocardial infarction. Exp Ther Med 6: 479-484, 2013.

3. Chan W, Stub D, Clark DJ, Ajani AE, Andrianopoulos N, Brennan AL, New G, Black A, Shaw JA, Reid CM, et al; Melbourne Interventional Group Investigators: Usefulness of transient and persistent no reflow to predict adverse clinical outcomes following percutaneous coronary intervention. Am J Cardiol 109: 478-485, 2012.

4. Svilaas T, Vlaar PJ, van der Horst IC, Diercks GF, de Smet BJ, van den Heuvel AF, Anthonio RL, Jessurun GA, Tan ES, Suurmeijer AJ and Zijlstra F: Thrombus aspiration during primary percutaneous coronary intervention. N Engl J Med 358: 557-567, 2008.

5. Berger PB and Ryan TJ: Inferior myocardial infarction. High-risk subgroups. Circulation 81: 401-411, 1990. 
6. Fu XH, Fan WZ, Gu XS, Wei YY, Jiang YF, Wu WL, Li SQ, Hao GZ, Wei QM and Xue L: Effect of intracoronary administration of anisodamine on slow reflow phenomenon following primary percutaneous coronary intervention in patients with acute myocardial infarction. Chin Med J (Engl) 120: 1226-1231, 2007.

7. Wang Y, Fu X, Wang X, Jia X, Gu X, Zhang J, Su J, Hao G, Jiang Y, Fan W, et al: Protective effects of anisodamine on renal function in patients with ST-segment elevation myocardial infarction undergoing primary percutaneous coronary intervention. Tohoku J Exp Med 224: 91-97, 2011.

8. Geng W, Fu XH, Gu XS, Wang YB, Wang XC, Li W, Jiang YF, Hao GZ, Fan WZ and Xue L: Preventive effects of anisodamine against contrast-induced nephropathy in type 2 diabetics with renal insufficiency undergoing coronary angiography or angioplasty. Chin Med J (Engl) 125: 3368-3372, 2012 .

9. Wang YB, Fu XH, Gu XS, Wang XC, Zhao YJ, Hao GZ, Jiang YF, Fan WZ, Wu WL, Li SQ and Xue L: Safety and efficacy of anisodamine on prevention of contrast induced nephropathy in patients with acute coronary syndrome. Chin Med J (Engl) 125: 1063-1067, 2012

10. Lee HC, An SG, Choi JH, Lee TK, Kim J, Kim JH, Chun KJ Hong TJ, Shin YW and Lee SK: Effect of intra-coronary nicorandi administration prior to reperfusion in acute ST segment elevation myocardial infarction. Circ J 72: 1425-1429, 2008.

11. Ota S, Nishikawa H, Takeuchi M, Nakajima K, Nakamura T, Okamoto S, Setsuda M, Makino K, Yamakado T and Nakano T: Impact of nicorandil to prevent reperfusion injury in patients with acute myocardial infarction: Sigmart Multicenter Angioplasty Revascularization Trial (SMART). Circ J 70: 1099-1104, 2006.

12. Ikeda N, Yasu T, Kubo N, Hashimoto S, Tsuruya Y, Fujii M, Kawakami $\mathrm{M}$ and Saito M: Nicorandil versus isosorbide dinitrate as adjunctive treatment to direct balloon angioplasty in acute myocardial infarction. Heart 90: 181-185, 2004.

13. TIMI Study Group: The Thrombolysis In Myocardial Infarction(TIMI) trial. Phase I findings. TIMI Study Group. N Engl J Med 312: 932-936, 1985.

14. Gibson CM, Cannon CP, Murphy SA, Ryan KA, Mesley R, Marble SJ, McCabe CH, Van De Werf F and Braunwald E: Relationship of TIMI myocardial perfusion grade to mortality after administration of thrombolytic drugs. Circulation 101 $125-130,2000$.

15. Kawai Y, Hisamatsu K, Matsubara H, Dan K, Akagi S, Miyaji K, Munemasa M, Fujimoto Y, Kusano KF and Ohe T: Intravenous administration of nicorandil immediately before percutaneous coronary intervention can prevent slow coronary flow phenomenon. Eur Heart J 30: 765-772, 2009.

16. Shen J, Zhang Q, Zhang RY, Zhang JS, Hu J, Yang ZK, Zheng AF, Zhang X and Shen WF: Clinical benefits of adjunctive tirofiban therapy in patients with acute ST-segment elevation myocardial infarction undergoing primary percutaneous coronary intervention. Coron Artery Dis 19: 271-277, 2008.
17. Huber K, Holmes DR Jr, van't Hof AW, Montalescot G, Aylward PE, Betriu GA, Widimsky P, Westerhout CM, Granger CB and Armstrong PW: Use of glycoprotein IIb/IIIa inhibitors in primary percutaneous coronary intervention: Insights from the APEX-AMI trial. Eur Heart J 31: 1708-1716, 2010.

18. Gibson CM, de Lemos JA, Murphy SA, et al; TIMI Study Group: Combination therapy with abciximab reduces angiographically evident thrombus in acute myocardial infarction: A TIMI 14 substudy. Circulation 103: 2550-2554, 2001

19. Rezkalla SH and Kloner RA: No-reflow phenomenon. Circulation 105: 656-662, 2002.

20. Reffelmann T and Kloner RA: The 'no-reflow' phenomenon: Basic science and clinical correlates. Heart 87: 162-168, 2002.

21. Galiuto L: Optimal therapeutic strategies in the setting of post-infarct no reflow: The need for a pathogenetic classification. Heart 90: 123-125, 2004.

22. Niccoli G, Lanza GA, Shaw S, Romagnoli E, Gioia D, Burzotta F, Trani C, Mazzari MA, Mongiardo R, De Vita M, et al: Endothelin-1 and acute myocardial infarction: A no-reflow mediator after successful percutaneous myocardial revascularization. Eur Heart J 27: 1793-1798, 2006.

23. Xiu RJ, Hammerschmidt DE, Coppo PA and Jacob HS: Anisodamine inhibits thromboxane synthesis, granulocyte aggregation, and platelet aggregation. A possible mechanism for its efficacy in bacteremic shock. JAMA 247: 1458-1460, 1982.

24. Zhao B, Li J, Luo X, Zhou Q, Chen H and Shi H: The role of von Willebrand factor and ADAMTS13 in the no-reflow phenomenon: After primary percutaneous coronary intervention. Tex Heart Inst J 38: 516-522, 2011.

25. Matsumoto H, Inoue N, Takaoka H, Hata K, Shinke T, Yoshikawa R, Masai H, Watanabe S, Ozawa T and Yokoyama M: Depletion of antioxidants is associated with no-reflow phenomenon in acute myocardial infarction. Clin Cardiol 27: 466-470, 2004.

26. Wang A, Chen F, Xie Y, Guo Z and Yu Y: Protective mechanism of nicorandil on rat myocardial ischemia-reperfusion. J Cardiovasc Med (Hagerstown) 13: 511-515, 2012.

27. Iwakura K: Modulation of individual susceptibility to the no-reflow phenomenon after acute myocardial infarction. Curr Pharm Des 19: 4519-4528, 2013.

28. Akai K, Wang Y, Sato K, Sekiguchi N, Sugimura A, Kumagai T, Komaru T, Kanatsuka H and Shirato K: Vasodilatory effect of nicorandil on coronary arterial microvessels: Its dependency on vessel size and the involvement of the ATP-sensitive potassium channels. J Cardiovasc Pharmacol 26: 541-547, 1995. 